\title{
Correction: ASPM promotes prostate cancer stemness and progression by augmenting Wnt-Dvl-3- $\beta$-catenin signaling
}

\author{
Vincent C. Pai ${ }^{1,3} \cdot$ Chung-Chi Hsu ${ }^{1,2} \cdot$ Tze-Sian Chan $^{1,2} \cdot$ Wen-Ying Liao ${ }^{3} \cdot$ Chih-Pin Chuu $^{4} \cdot$ Wei-Yu Chen ${ }^{5}$. \\ Chi-Rong $\mathrm{Li}^{6} \cdot$ Ching-Yu Lin ${ }^{4} \cdot$ Shu-Pin Huang ${ }^{7} \cdot \mathrm{Li}^{-}$Tzong Chen ${ }^{3} \cdot$ Kelvin K. Tsai ${ }^{1,2,3}$
}

Published online: 2 November 2018

(c) Springer Nature Limited 2018

Correction to: Oncogene https://doi.org/10.1038/s41388018-0497-4; published online: 28 Sep 2018

In the published version of this paper the author Shu-Pin Huang's surname was incorrectly given as Hwang instead of Huang. This has now been corrected in the HTML and PDF versions of the paper.
Kelvin K. Tsai

tsaik@tmu.edu.tw

1 Graduate Institute of Clinical Medicine, College of Medicine, Taipei Medical University, Taipei City 11031, Taiwan

2 Division of Gastroenterology, Department of Internal Medicine, Laboratory of Advanced Molecular Therapeutics, Wan Fang Hospital, Taipei Medical University, Taipei City 11696, Taiwan

3 National Institute of Cancer Research, National Health Research Institutes, Zhunan, Miaoli County 35053, Taiwan
4 Institute of Cellular and System Medicine, National Health Research Institutes, Zhunan, Miaoli County 35053, Taiwan

5 Department of Pathology, Wan Fang Hospital, Taipei Medical University, Taipei City 11696, Taiwan

6 Department of Teaching and Research, Taichung Hospital Ministry of Health and Welfare, Taichung City 40343, Taiwan

7 Department of Urology, Kaohsiung Medical University Chung-Ho Memorial Hospital, Kaohsiung City 80756, Taiwan 